Revista Calidad en la Educación Superior

Programa de Autoevaluación Académica

Universidad Estatal a Distancia, Costa Rica

ISSN 1659-4703

revistacalidad@uned.ac.cr

\title{
MODELO PARA LA INVESTIGACIÓN COMPARATIVA ENTRE UNIVERSIDADES DE EDUCACIÓN A DISTANCIA, ABIERTAS O EN LÍNEA
}

\section{A MODEL FOR COMPARATIVE RESEARCH AMONG OPEN, ONLINE AND DISTANCE EDUCATION UNIVERSITIES}

\author{
Alexandra María Abarca Chinchilla ${ }^{1}$ \\ aleabarca@uned.ac.cr \\ Jennifer Azofeifa Retana ${ }^{2}$ \\ jazofeifa@uned.ac.cr \\ Ana Cristina Brenes Villalobos ${ }^{3}$ \\ abrenes@uned.ac.cr \\ Universidad Estatal a Distancia, Costa Rica
}

\author{
Volumen 7, Número 1 \\ Mayo 2016 \\ pp. $252-283$
}

Recibido: 29 de enero, 2016

Aprobado: 30 de abril, 2016

\footnotetext{
${ }^{1}$ Bach Alexandra María Abarca Chinchilla, del Programa de Investigación en Fundamentos de Educación a Distancia (PROIFED). Correo electrónico: aleabarca@uned.ac.cr

2 Licda Jennifer Azofeifa Retana, del Programa de Investigación en Fundamentos de Educación a Distancia (PROIFED). Correo electrónico: jazofeifa@uned.ac.cr

3 Mag. Ana Cristina Brenes Villalobos, del Programa de Investigación en Fundamentos de Educación a Distancia (PROIFED). Correo electrónico: abrenes@uned.ac.cr
} 


\section{Resumen}

Dada la necesidad de contar con un modelo que supere los principales vacíos que, a nivel histórico-metodológico, se han identificado en estudios comparativos de educación a distancia y que vayan más allá de la mera descripción de situaciones o contextos educativos; en el Programa de Investigación en Fundamentos de Educación a Distancia (PROIFED) surge el interés de proponer un modelo para realizar este tipo de estudios, con el fin de desarrollarlos en redes flexibles de profesionales, que promuevan el intercambio de información para conocer cómo otras universidades dirigen y resuelven sus situaciones de conflicto y cómo proyectan su quehacer en la educación a distancia, abierta o en línea. Se dispone el presente modelo, el cual procura ser integrador y articulado; abierto o adaptativo a algunos requerimientos específicos que la persona investigadora requiera, con el fin de fortalecer y promover el mejoramiento del modelo de educación a distancia de la Universidad Estatal a Distancia (UNED), Costa Rica.

Palabras clave: Educación a distancia, Educación en línea, Educación abierta, Educología comparativa, Estudios comparativos, Red de profesionales.

\section{Abstract}

The Research Program on Fundamentals of Distance Education of UNED Costa Rica proposes an analytical model for the generation of comparative studies among open, online and distance education universities and institutions. This model is intended to satisfy a historical and methodological vacuum identified in comparative studies that are usually limited to the mere descriptions of educational contexts and situations. The comparative studies proposed by this model, on the other hand, are thought to be developed by professionals assembled in flexible networks and to promote the exchange of information about the ways in which open, online and distance education universities confront and resolve their situational conflicts and how these universities project their work. This model for comparative studies is designed to be open, adaptable to specific requirements of researchers, and to strengthen and promote the improvement of the distance education model of UNED Costa Rica.

Keywords: Distance learning, Online education, Open education, Comparative educology, Comparative studies, Professional networking.

\section{Introducción}

El presente proyecto pretende elaborar y validar un modelo para comparar diferentes sistemas de educación superior bajo la modalidad de educación a distancia, entre universidades de educación a distancia, abiertas o en línea. 
Esto constituye una necesidad para el PROIFED, ya que es fundamental conocer el estado de aspectos de gestión, organizacionales, educativos, tecnológicos, entre otros, de universidades pares; así como conocer los retos que estas enfrentan y la forma en que los han superado, para enriquecer el quehacer institucional de la UNED.

Al respecto, el PROIFED establece en su plan operativo anual, el desarrollo de proyectos de investigación en modelos de organización de instituciones de educación a distancia, por lo que la UNED estaría apostando a liderar redes internacionales para la innovación de esta modalidad educativa.

\section{Justificación}

La construcción de un modelo que permita realizar estudios comparativos en instituciones de educación superior a distancia, abierta o en línea que complete los vacíos que a nivel históricometodológico, se han identificado, tal como sugiere Olivera (2011) en relación con algunos estudios de educología comparativa en los que "prevalece cierta confusión, pese a ideas claras e información precisa, porque carecen de un "modelo" o estructura general ordenadora" ( $p$. 314). El modelo podría fundamentar estudios que vayan más allá de la mera descripción de situaciones o contextos educativos. Este prototipo brindará al usuario la posibilidad de desarrollar, entre redes flexibles de profesionales, el intercambio de información y conocer cómo otras universidades dirigen y proyectan su quehacer en la educación a distancia, abierta o en línea.

Dicho modelo se basa en el enfoque comparativo o educología comparativa que, según Olivera (2011),

investiga los fenómenos educativos globales o particulares que se dan en dos o más sociedades o grupos humanos, en cuanto a las relaciones de semejanza y diferencia que existen entre ellos, y entre las formas de interacción que mantienen con sus respectivos entornos sociales, a fin de contribuir el conocimiento y la comprensión de dichos fenómenos educativos, propios y ajenos y de enriquecer los procesos de toma de decisiones para su mejoramiento (p. 34). 
Esta forma de investigación, agrega este autor, se puede desarrollar mediante estudios de casos, ya que son parte de la educología comparativa, en tanto sirvan a la comparación de fenómenos o sistemas que exponen dos o más realidades educativas usando el mismo esquema de análisis.

A partir de lo anterior, la presente investigación se basa en la propuesta de Olivera (2011), con el fin de no reincidir en algunas de las principales deficiencias que, según Saba (2014), tienen las investigaciones en este ámbito; a saber:

- La comparación de modelos educativos que son fundamentalmente distintos entre sí.

- La incorrecta elaboración de constructos que no permiten la comparación real de criterios.

- La invisibilización de aspectos particulares sobre la generalización de los resultados.

- La descontextualización de los sujetos de información.

- El desconocimiento de aspectos históricos que hace que las investigaciones de este tipo se enclaustren en un lapso determinado.

Por ello, la finalidad de este modelo será contextualizar los estudios a las particularidades o condiciones, tanto internas como externas, de las universidades por comparar y de las personas investigadoras, de acuerdo con sus intereses.

\section{Metodología}

La comparación es un ejercicio mental intuitivo al que recurre constantemente el individuo; primeramente, para formar conceptos e ideas $y$, en un segundo esfuerzo mental, para establecer relaciones de semejanza y diferencia entre elementos. Este procedimiento permite confrontar, constituir categorizaciones, tipologías, sistematizaciones; por lo que, tal y como menciona Olivera (2011), es parte del origen mismo del conocimiento.

Como metodología de investigación, la comparación en espacios educativos se sustenta en la educología comparativa, la cual es entendida como la disciplina científica que busca, mediante el uso de la metodología comparativa, establecer relaciones entre los fenómenos educativos 
que se puedan presentar, a partir de la variedad de quehaceres que implican la ejecución y puesta en marcha de los sistemas o las instituciones educativas en dos o más sociedades 0 grupos humanos.

Esta disciplina estudia también la interacción de estos fenómenos con sus respectivos entornos sociales; a su vez, permite mediante la utilización de diferentes instrumentos y enfoques, confrontar realidades educativas similares por medio de la detección de las semejanzas que permiten generalizaciones; o bien, reconocer las diferencias particulares que identifican cada sistema.

En las ciencias sociales, el proceso comparativo como parte del método de investigación, tendrá que ir a un nivel superior, a un espacio de mayor abstracción; es decir, en este ámbito del conocimiento, la comparación aplica cuando se trata de conjuntos ya elaborados de conceptos teóricos que refieren a realidades de tipo similar y que se desarrollan en grupos sociales diferentes, ya sean países, zonas, regiones o a nivel global.

En el ámbito educativo, esta posibilidad de comparar se convierte en una exigencia para intentar construir generalizaciones válidas y un aporte significativo al conocimiento mismo. Igualmente, en la educación, la validez de una investigación comparativa está fundamentada en la información que se pueda obtener de los objetos materiales que se desean comparar; sin embargo, es preciso que esta información se obtenga de tres conjuntos de datos:

- Io interno: de lo que sucede dentro de cada sistema educativo;

- desde fuera: el entorno que condiciona en gran medida al sistema;

- la vinculación de ambos: o sea, de las relaciones que se dan entre el entorno y el sistema.

La articulación de estos datos permite realizar una descripción y análisis reticulado y, simultáneamente, por su condición comparativa, utilizarse para encontrar relaciones de semejanza y diferencia tanto entre los componentes estructurales como entre las formas de interacción de las instituciones educativas con sus respectivos entornos. 


\subsection{Objetivos}

Este informe tiene como objetivo general confeccionar un modelo para la elaboración de estudios comparativos de universidades de educación a distancia, abiertas o en línea, que permita el mejoramiento del sistema de gestión de Educación a Distancia de la UNED; y como objetivos específicos:

1. Indagar sobre los distintos enfoques metodológicos para la elaboración de estudios comparativos en educación.

2. Identificar constructos que permitan establecer relaciones de semejanza y diferencia entre elementos funcionales y operativos de universidades de educación a distancia, abiertas o en línea.

3. Proponer un modelo para la realización de estudios comparativos entre universidades de educación a distancia, abiertas o en línea.

\section{La educación superior a distancia}

Existen diversas posiciones teóricas en relación con la definición del concepto sobre educación a distancia, siendo igualmente estos válidos e importantes, se considera que el siguiente se apega efectivamente a la descripción de la realidad del sistema educativo de la UNED. Según consulta realizada al tesauro de la UNESCO (2015a) la educación es un:

proceso mediante el cual las sociedades transmiten en forma intencionada el acervo de información, comprensión, conocimientos, actitudes, valores, habilidades, competencias y comportamientos de una generación a otra. Comprende el uso de actividades de comunicación destinadas a producir aprendizaje. (sección Educación, párr. 12).

De acuerdo con la anterior definición, la educación superior se entiende, entonces, como otro escalafón que los seres humanos deben superar en su formación académica, teniendo claro que esta tiene por objetivo su profesionalización y especialización. 
A través de los años, la educación superior ha utilizado diferentes medios para llegar a más personas alrededor del mundo, y es en este acoplamiento que la educación superior a distancia se ha convertido en una opción que permite el acceso a personas que, por diferentes razones, no pueden asistir a sistemas educativos presenciales.

El debate en torno al modelo de educación superior a distancia se ha centrado en la investigación sobre aspectos meramente pedagógicos y didácticos de los procesos de aprendizaje, sus actores (estudiantes y docentes) así como la inserción de las Tecnologías de la Información y la Comunicación (TIC); no obstante, se han dejado de lado elementos sustanciales y trascendentales de gestión y organización, los cuales han de revisarse constantemente, si se pretende mejorar el servicio que las instituciones educativas que siguen este modelo le brindan a la sociedad.

Actualmente, las universidades de educación a distancia se valen, entre otras herramientas, de las TIC, las cuales se han utilizado para la mediación del aprendizaje. Continuando con el referente UNESCO (2015b), la educación a distancia habilitada por las TIC (aparatos de radio, computadoras personales, internet, recursos audiovisuales o impresos en un grado mínimo) es aquella que imparte "todo o una porción significativa de la enseñanza al estudiantado que se encuentran en otro espacio físico y en otro momento en cuanto al tiempo". (sección Educación a distancia, párr. 3).

Así, la educación a distancia puede adoptar una variedad de formas. Algunas definidas por la UNESCO (2015b) son: 
Tabla 1

Algunas formas de educación a distancia según la TIC habilitada.

\begin{tabular}{|l|l|}
\hline $\begin{array}{l}\text { Formas de educación a } \\
\text { distancia: }\end{array}$ & \multicolumn{1}{|c|}{ TIC habilitada: } \\
\hline $\begin{array}{l}\text { Aprendizaje a distancia } \\
\text { basado en internet. }\end{array}$ & Ya sea en forma sincrónica o asincrónica. \\
\hline $\begin{array}{l}\text { Tele-curso o educación } \\
\text { basada en transmisiones. }\end{array}$ & El contenido es impartido vía radio o televisión. \\
\hline $\begin{array}{l}\text { Enseñanza basada en cd } \\
\text { o dvd-rom para auto- } \\
\text { aprendizaje. }\end{array}$ & $\begin{array}{l}\text { El estudiante interactúa con el contenido computacional } \\
\text { guardado en un cd o un dvd-rom. }\end{array}$ \\
\hline $\begin{array}{l}\text { Aprendizaje basado en } \\
\text { dispositivos móviles. }\end{array}$ & $\begin{array}{l}\text { El estudiante accede a los contenidos del curso que están } \\
\text { guardados en un dispositivo móvil o a través de un servidor } \\
\text { inalámbrico. }\end{array}$ \\
\hline $\begin{array}{l}\text { Aprendizaje a distancia } \\
\text { integrado. }\end{array}$ & $\begin{array}{l}\text { Combina modalidades en vivo frente a las modalidades } \\
\text { grabadas, ofrece interacción individualizada frente a } \\
\text { enseñanza en grupo a través de varios canales y/o } \\
\text { materiales impresos que son utilizados en un grado mínimo, } \\
\text { etc. Esta modalidad excluye el aprendizaje a distancia } \\
\text { basado en correspondencia que se lleva a cabo } \\
\text { exclusivamente mediante correo postal regular. }\end{array}$ \\
\hline
\end{tabular}

Fuente: elaboración propia.

Muchos autores han definido la educación a distancia, ya que en su evolución ha sido entendida de distintas maneras de acuerdo con factores como la zona geográfica, la institución, el tipo de estudiantes al que va dirigido o la época que se evoca. Para ejemplificar esta variedad de caracterizaciones de la educación a distancia, se consignan algunas en la siguiente tabla, basándose en las reseñas propuestas por Lobo y Fallas (2008) 
Tabla 2

Algunas reseñas de educación a distancia

\begin{tabular}{|c|c|c|c|}
\hline Autor & Año & Reseña & Puntos convergentes \\
\hline Alonso & 2006 & $\begin{array}{l}\text { Posibilita la educación formal y no } \\
\text { formal a lugares remotos y sectores } \\
\text { con limitaciones de tiempo. }\end{array}$ & $\begin{array}{l}\text { - } \\
\text { - Accesibilidad }\end{array}$ \\
\hline Marchessou & 2001 & $\begin{array}{l}\text { La enseñanza es dirigida por alguien } \\
\text { distante en tiempo espacio del } \\
\text { aprendiz. }\end{array}$ & $\begin{array}{l}\text { remota. } \\
\text { - Asincrónica. } \\
\text { - Uso de TIC. }\end{array}$ \\
\hline Guimarães & 1996 & $\begin{array}{l}\text { Difiere de la enseñanza presencial en } \\
\text { metodología de enseñanza, la } \\
\text { estructura organizacional y el orden } \\
\text { económico. }\end{array}$ & $\begin{array}{l}\text { - Aprendizaje } \\
\text { independiente. } \\
\text { - Enseñanza } \\
\text { controlada. }\end{array}$ \\
\hline B. Hölmberg & 1985 & $\begin{array}{l}\text { Se basa en la comunicación no } \\
\text { directa; representado por otros } \\
\text { medios diferentes la palabra escrita. }\end{array}$ & $\begin{array}{l}\text { - Autonomía. } \\
\text { - Orientación } \\
\text { docente. }\end{array}$ \\
\hline M. Moore & 1972 & $\begin{array}{l}\text { Método de instrucción en que las } \\
\text { conductas docentes acontecen } \\
\text { aparte de las discentes. }\end{array}$ & \\
\hline
\end{tabular}

Fuente: elaboración propia.

Actualmente, se puede destacar la caracterización de educación a distancia con los aportes expresado por Moreno (2012)

la educación a distancia como una estrategia organizacional, metodológica y tecnológica para establecer la comunicación entre quienes participan en un proceso educativo, los contenidos que se requieren en dicho proceso y las instituciones responsables, aunque todos esos elementos no coincidan en tiempo y lugar; en el entendido de que más importante que superar las distancias geográficas es salvar las distancias sociales, económicas y culturales. (p.17) 
Por su parte, García (2014) indica que, cuando se habla de educación a distancia, se hace referencia a un ámbito, cuyas características son las siguientes:

- Emergente: emergen nuevas posibilidades, como conceptos, estructuras, etc.

- Complementario: se complementa a los esquemas presenciales.

- Complejo: intervienen diferentes agentes, profesores, aula, estudiantes.

- Problemático: surgen de filosofías e ideas muy diferentes.

- Heterogéneo: tiene diversidad de propuestas, formulaciones y tipos.

- Con bases múltiples: se basa en una gran cantidad de ciencias y conocimientos.

A pesar de lo anterior, y como punto de convergencia entre los autores anteriormente mencionados, García (2014, p. 47) concluye con una definición un tanto más abarcadora de la educación a distancia en la que dice que

la enseñanza a distancia es un sistema tecnológico de comunicación bidireccional (multireccional), que puede ser masivo, basado en la acción sistemática y conjunta de recursos didácticos y el apoyo de una organización y tutoría, que, separados físicamente de los estudiantes, propician en estos un aprendizaje independiente y cooperativo.

Este sistema, además, se caracteriza por:

- Separación: hay una separación física entre docentes-estudiantes y de los propios estudiantes entre sí.

- Estudio independiente: el estudiantado controla el tiempo, el espacio, determinados ritmos de estudio y, en algunos casos, itinerarios, actividades y tiempos de evaluación.

- Comunicación e interacción: la comunicación (estudiante-contenidos, estudiantedocente y entre estudiantes) se desarrolla de manera mediada, sincrónica o asincrónicamente, a través de los diferentes medios y recursos tecnológicos.

- Institución soporte: se cuenta con una organización que planifica, diseña, produce materiales (por sí misma o por encargo), realiza el seguimiento y brinda la motivación del proceso de aprendizaje mediante la tutoría, evalúa y acredita los aprendizajes.

Como ya se evidenció, la educación a distancia ha sido definida desde diferentes aristas; sin embargo, todas las definiciones tienen aspectos comunes entre sí. 
En este punto, es importante indicar la diferenciación entre educación a distancia y educación abierta, ya que tradicionalmente estas se han visto como sinónimos, a pesar de que no lo son: la educación abierta se refiere a la eliminación de requisitos y de barreras para el acceso al aprendizaje, de lo cual se desprende que no toda educación a distancia es abierta (ni toda educación abierta es a distancia). Así, si bien la educación abierta puede desarrollarse a distancia, se diferencia de esta en el hecho de que, como ya se indicó, no establece requisitos, principalmente de ingreso, por lo que su estructura tiende a ser más flexible. No obstante lo anterior, existen universidades que son de educación a distancia y abiertas, como por ejemplo la UNED de Costa Rica.

Otra forma de educación a distancia es la educación en línea (online) que también se conoce como 'educación virtual', 'formación en espacios virtuales', 'e-learning', entre otros. En estos casos, el énfasis radica en que tanto los materiales de estudio como la relación entre docentes y estudiantes se realiza exclusivamente a través de las TIC.

\section{Propuesta de modelo comparativo}

En primer lugar, interesa definir lo que se entiende por la palabra 'modelo', y en este sentido, Entre los distintos significados y usos del término modelo (que proviene del italiano modello), se destaca la referencia al arquetipo o punto de referencia para su imitación o reproducción [...] Un modelo también es el esquema teórico de un sistema o de una realidad compleja" (Definición.DE, 2015, párr. 1).

En el Diccionario de la Lengua Española (2014) se define la palabra modelo como: "1. m. Arquetipo o punto de referencia para imitarlo o reproducirlo". (párr. 1)

Asimismo, González, Nieto, Portela y Martínez. (2012, p. 4) lo puntualizan como:

[...] un tipo de elaboración conceptual intermedio que conecta una teoría en el objeto o realidad que esta estudia. Cada modelo delimita algunas dimensiones de un fenómeno, así como relaciones entre las mismas con el propósito de orientar y aportar conocimiento a la teoría. 
Para Olivera (2011, p. 141),

Un modelo es la combinación estructurada de un número más o menos elevado de variables (entre las que puede haber "tipos"), que, o bien pretende representar esquemáticamente la realidad mediante relaciones análogas a las que se han observado en ésta (...) o bien, es una reconstrucción simbólica y lógica de la realidad...

Continúa este autor, los modelos teóricos "permiten profundizar el estudio de realidades complejas y manipularlas mentalmente; luego la experimentación o la observación controlada permitirá confrontar, o sea, comparar el comportamiento del modelo con el de la realidad o viceversa" (p.142).

En las estructuras formales de las organizaciones es notable entender la existencia de modelos que proporcionan sentido y se convierten en el fundamento de las instituciones, con las particularidades que los distinguen e identifican. Esta base, aunque no sea fácil de comprender en un primer momento por la complejidad que logra alcanzar, ofrece una gama de componentes por valorar entre similares, lo que permite establecer relaciones de comparación.

Otro referente de modelo que no se debe obviar son los modelos mentales que, según Senge (2011, p. 17), son definidos como "supuestos hondamente arraigados, generalizaciones e imágenes que influyen sobre nuestro modo de comprender el mundo y actuar". Estos modelos mentales interiorizados por actores en los diferentes ámbitos organizacionales, se logran identificar y plasmar junto con los modelos formales de las organizaciones, lo que conlleva a descubrir un gran número de posibilidades de cómo funcionan estas comparativamente.

El modelo comparativo que se plantea en esta propuesta permitirá ahondar información pertinente, para validar o superar situaciones de mejora en la UNED y proponer un quehacer profesional más holístico. El modelo, además, procura ser integrador y articulado; abierto o adaptativo a algunos requerimientos específicos que la persona investigadora demande. A su vez, facilitará la identificación de aportes significativos en el plano de la conducción de los sistemas de educación superior bajo la modalidad a distancia, abierta o en línea; tomando en cuenta que no es una práctica usual, como labor investigativa, que se compartan o analicen 
modelos de gestión institucional, pues la mayoría de los esfuerzos resuelven principalmente el acto educativo.

El aspecto anterior es confirmado por Zawacki-Richter y Anderson (2014), quienes indican que de las tres grandes categorías de estudio sobre la educación a distancia, de la que se encuentra mayor cantidad de información e investigación es de la referente al nivel micro, condicionada a los procesos de enseñanza-aprendizaje; en tanto que los otros dos niveles, macro y meso, son los menos abordados por académicos en esta disciplina.

En este sentido, esta propuesta de investigación se enmarca en el nivel meso, circunscrito a gestión, organización y tecnología de la educación a distancia; y en menor medida al nivel macro, pues se tiene el interés de coadyuvar en el entendimiento de los sistemas y las teorías de educación a distancia mediante la investigación y la transferencia de conocimiento.

A continuación, se indican los aspectos que componen el modelo:

1. Universidad: la base comparativa y central del modelo será la institución de educación superior, conocida como universidad, cuya modalidad educativa será a distancia, abierta o en línea. Esta tendrá dos dimensiones: la externa y la interna.

\subsection{Dimensión externa}

\subsubsection{El contexto social}

Pretende describir y analizar el entorno físico de cada una de las instituciones educativas por investigar. Está dirigido a conocer la ubicación física de las universidades a comparar; los contextos socioeconómicos, culturales y educativos de la sociedad que sustenta la universidad a distancia, abierta o en línea. En esta sección también se da un acercamiento a la política educativa que promulga la administración gubernamental, en una tentativa por conocer lo que condiciona externamente a las instituciones educativas.

\subsection{Dimensión interna}


Compuesta por dos grandes apartados abarcadores que describen el escenario interno de la universidad; a saber, la caracterización y la planificación institucional.

\subsubsection{Caracterización institucional}

Segmento del modelo que permite realizar una descripción profunda de la realidad particular de la institución de educación superior. Refiere a la razón filosófica- epistemológica de su quehacer educativo, así como a las políticas y regulaciones que rigen su gestión y accionar. Los componentes de este apartado serán normativa institucional, modelo pedagógico y tipo de institución. Seguidamente, se describirá cada uno:

\subsubsection{Normativa institucional}

La normativa institucional es el sustento legal por medio del cual una institución logra regular la convivencia de los diferentes actores y sus acciones. Constituye una serie de enunciados considerados base para fundamentar las buenas prácticas en los ámbitos académico y administrativo, y acuerpados en leyes, estatutos, reglamentos, normas y procedimientos.

\subsubsection{Modelo pedagógico}

Un modelo pedagógico es un conjunto de elementos que intervienen en una situación educativa determinada, de modo que preside y orienta claramente la forma en que esta debe llevarse a la práctica. Describe quehaceres como la docencia, el diseño curricular, el ambiente educativo, el proceso pedagógico, la producción de materiales para el aprendizaje y los procesos de evaluación entre otros; todos ellos, con la finalidad de ofrecer una formación de calidad al estudiante.

Tanto los principios pedagógicos como las corrientes pedagógicas y las diferentes variables de proceso de enseñanza-aprendizaje inspiran a los docentes y pedagogos a proponer, experimentar o ensayar diferentes formas de organizar este proceso. Esto ha generado diversos modelos para la organización, ejecución y planificación del aprendizaje y la enseñanza; así como diversas clasificaciones o disposiciones.

García (2014) sugiere para la educación a distancia varios modelos pedagógicos: 
- Tradicional. Autoritario y conservador. Con una estructura vertical y normativa; hace uso de tecnología sofisticada. En este, la habilidad memorística del estudiante se destaca entre sus destrezas para alcanzar el éxito formativo.

- Participativo. El aprendiente es el centro y el protagonista del proceso. El docente es facilitador y orientador. Se basa en la interacción entre actores y entre estos y el medio.

- Conductista. Muy utilizado aún en la actualidad, afirmado en la pedagogía por objetivos. Estructurado rígida y jerárquicamente, y organizado de forma meticulosa, previsoria y ordenada. El conocimiento se adquiere con el alcance y consecución de objetivos operativos.

- Constructivista. Se enfatiza en los procedimientos y estrategias cognitivas que, llevan al estudiante, a través de su propia intervención y participación, a la adquisición del conocimiento y a la construcción de nuevos significados. El docente es tanto mediador del proceso educativo, como programador, organizador y faciltador.

- Centrado en el docente (magistrocentrismo). Se enfoca en la actividad docente de enseñar, pero vista como una transmisión de contenidos. Da importancia al uso de los materiales y la tecnología. Se adapta fácilmente al e-lerning.

- Centrado en el saber (logocentrismo). Concentra sus objetivos en la mayor cantidad de contenidos que el estudiante recibe del profesor o autor del material, y los cuales debe dominar generalmente por medio de la memoria.

- Centrado en el estudiante (paidocentrismo). Ubica al estudiante como protagonista de su propia formación. Los estilos propios y los ritmos particulares de aprendizaje se respetan para convertir al docente en un facilitador de los aprendizajes. Se yuxtapone idealmente con los principios constructivistas del aprendizaje. Este modelo se engrana de manera asertiva con la educación a distancia.

- Centrado en las tecnologías (tecnocentrismo). Destaca la tecnología por encima de los otros componentes del proceso, incluyendo los actores. El docente es un proveedor de información y el estudiante un usuario que tiene acceso a los contenidos cuando y donde lo disponga. Presupone el uso de las últimas tendencias tecnológicas, por lo que se le da poca importancia al proceso pedagógico y los resultados.

- Centrado en las interacciones (interaccionismo). Tiene una estrecha relación con el anterior, pero con una perspectiva constructivista. Aprovecha las tecnologías 
colaborativas para establecer relaciones sincrónicas y asincrónicas entre actores. El aprendizaje se da en el intercambio de ideas, el consenso, y la construcción social, tomando como base las TIC.

- Integrador. Basado en un buen diseño pedagógico, contenidos adaptados al curso e integrados a una guía didáctica efectiva que permita la búsqueda, el análisis, la selección y el procesamiento de los contenidos que el estudiante habrá de construir según su ritmo de aprendizaje.

Claro está que cada institución o sistema adaptará su modelo pedagógico a sus contextos, intereses, funciones o necesidades.

\subsubsection{Tipo de institución}

Es el componente que tipifica a la organización de acuerdo con la forma en que gestiona administrativa y operativamente su quehacer educativo. Permite ahondar en el sistema institucional para conocer sus características, condiciones, programas y proyectos, cobertura, uso de las TIC, entre otros aspectos.

García (2004) propone una tipología para clasificar las instituciones de educación a distancia, en la cual destacan tres grandes categorías: "unimodales, duales o bimodales y según la procedencia de los programas ofrecidos" (p. 6). A continuación, se presenta la descripción de cada una:

\section{- Instituciones unimodales}

Los modelos institucionales unimodales o autónomos son los que disponen únicamente de la metodología de enseñanza-aprendizaje a distancia; indistintamente del tipo de recursos que empleen para el soporte de los contenidos y para la comunicación. Dentro de este modelo se incluyen las tipologías de interacción y comunicación como las siguientes: sincronía, asincronía, virtualización total o parcial, tutorías presenciales (en los casos que se requieran), tutorías virtuales, etc. Este tipo de modelo puede subdividirse en:

- A distancia sin servicios virtuales: instituciones de educación a distancia tradicional. 
- A distancia con servicios virtuales: instituciones de educación a distancia tradicional que poco a poco han ido incorporando los sistemas electrónicos.

- Virtuales autónomas: instituciones en las que todos los servicios que se ofrecen son virtuales.

- Virtuales dependientes: instituciones que ofrecen cursos exclusivamente virtuales de educación no formal (capacitación, actualización profesional), producidos por otra organización.

\section{- Instituciones duales o bimodales}

Para García (2004, p.7), "las instituciones duales, bimodales, mixtas o de enseñanzas combinadas, también denominadas por algunos como parcialmente a distancia", disponen de los dos modelos clásicos: el presencial, que atiende a los alumnos que acuden a sus aulas, bibliotecas y laboratorios, con la metodología habitual; y el de a distancia, que implica que, dentro del mismo centro o institución, existen alumnos que siguen los estudios a través de esta modalidad, total o parcialmente. Este tipo de modelo puede subdividirse en:

- Estudios presenciales y a distancia: algunos programas se imparten solo a distancia o bien, combinan la educación a distancia con la modalidad presencial.

- Semipresencial: son estudios en los que cada asignatura o materia cuenta con un determinado porcentaje de tiempo lectivo desarrollado presencialmente y el resto, a distancia. Se le conoce como blended learning o blended e-learning.

- Algunas materias: es mixto. Algunas materias o asignaturas se ofrecen en formato presencial y el resto, plenamente a distancia.

- La misma infraestructura: los recursos docentes, de espacios y servicios de la educación presencial se ponen al servicio de los estudiantes a distancia.

- El complemento virtual: son todas aquellas instituciones presenciales que ofrecen servicios virtuales complementarios a sus estudiantes.

\section{- Instituciones de acuerdo con la procedencia de los programas ofrecidos}


Según García (2004), son universidades que han ido incorporando progresivamente los estudios a distancia o que ya nacieron con esa vocación fundacional. Hanna, citado por García (2004), menciona que se pueden encontrar cuatro tipos en la actualidad:

- Programas a distancia: son programas universitarios, ofrecidos por universidades unimodales y bimodales, que aprovechan todas las tecnologías para ser ofrecidos en formato virtual.

- Programas de universidades corporativas: acreditan la capacitación de sus propios recursos humanos en servicio y de aquellos que pueden formar parte de ellas posteriormente.

- Programas de universidades con vocación internacional: son programas universitarios que, por sí mismos o mediante convenios con otras instituciones, ofrecen programas de estudio, de acreditación o certificación internacional, en su mayoría posgrados; ya sea que el estudiante resida fuera del país en el que se encuentra la universidad o bien, que reciba un apoyo local de esta universidad en su propio país. Otras opciones son que el programa de estudios que el estudiante esté recibiendo sea proporcionado por una universidad distinta a la que está certificando, que se trate de programas gemelos (el mismo programa en diferentes universidades), y por último, que sean desarrollados por varias instituciones, ya sean externas o internas a un país en el que se determina la responsabilidad de cada una de estas en el desarrollo y certificación del programa.

- De alianzas entre universidades y empresas: son alianzas, convenios y asociaciones estratégicas entre diferentes empresas con alguna universidad, con el fin de ofrecer determinados estudios o servicios mediante la modalidad a distancia.

\subsubsection{Planificación institucional}

Es el segundo apartado de la dimensión interna de las universidades. Este se refiere a que tanto la educación abierta, como a distancia y en línea, requieren de la planificación y la organización institucional para lograr el éxito. En esta se incluyen asuntos de interés político, social y económico, los cuales inciden en los planes, programas y proyectos de la gestión educativa. 
Las múltiples funciones consideradas en el proceso educativo, desde la descripción de los objetivos de los programas, la población meta, el diseño curricular, los contenidos, el diseño instruccional, los procesos de matrícula de los estudiantes, las tutorías, los mecanismos de apoyo a los estudiantes, la evaluación de los aprendizajes, la titulación, la producción de los materiales, el financiamiento y distribución de los recursos; hasta la evaluación del desempeño individual, por mencionar algunos, son quehaceres institucionales susceptibles a la planificación.

La planificación ha de verse como una herramienta de gestión que permite fundamentar la toma de decisiones de las organizaciones en relación con el quehacer actual de esta y dirige el camino que deberá recorrer en el futuro para ajustarse a las demandas y cambios que asigna el entorno; esto con el fin de alcanzar mayor eficacia, eficiencia y calidad en los servicios y productos que la organización ofrece.

Desde esta dimensión se describen el marco estratégico institucional y la gestión institucional como elementos que generan información importante para establecer las relaciones de semejanza y diferencia, así como los patrones de estas relaciones.

\subsubsection{Marco estratégico institucional}

La planificación se vuelve estratégica desde el momento en el que se desarrolla en concordancia con el marco estratégico de la institución educativa, entendido este como el fundamento filosófico-epistemológico de la organización, el cual está compuesto por la visión, la misión y los valores de la universidad.

El marco estratégico tiene como fin el ordenamiento y la declaratoria de los objetivos institucionales, que son la base de la elaboración de planes organizacionales que se derivan, directa y exclusivamente, de la declaración de la misión institucional y de los análisis prospectivos del contexto. Además, los objetivos institucionales sirven de referencia para la formulación de políticas y estrategias institucionales, y estos se convierten en las metas o propósitos que la institución debería lograr en forma permanente y continua. 
Los componentes del marco estratégico se describen a continuación:

\section{- Misión}

Es la razón que evidencia la existencia de la institución. Esta delimita el marco y el espacio para asignar y comprometer los principales recursos, principios, valores, expectativas y esfuerzos creativos de los individuos en la institución. Al respecto, David (2008) sostiene que la misión define lo que es la institución. Para este autor, es el elemento que distingue a una organización de otras que ofrecen o podrían ofrecer el mismo servicio. Igualmente, la misión sirve de recuadro para la evaluación institucional futura y permite, tanto a la comunidad externa al centro académico como a lo interno de esta, la comprensión de lo que debería hacer la institución.

\section{- Visión}

Es la meta que pretende alcanzar la organización por medio de los objetivos y sus estrategias.

\section{- Valores}

Una vez que se describen los elementos anteriores, es necesario reconocer los valores corporativos o valores institucionales que impulsan las relaciones y esfuerzos humanos, con el fin de crear un ambiente conveniente y fructuoso para el alcance de los objetivos. Estos valores consisten en un acervo de pautas de comportamiento y creencias fundamentales que pueden considerarse en cierta forma la "cosmovisión" que tienen los individuos, pero en el plano organizacional. Relata el conjunto de creencias sobre las cuales se instauran las premisas de todas las actuaciones y normas y comprometen oficialmente a los individuos que laboran en la institución.

\subsubsection{Gestión institucional}

El término 'gestión' se entiende como un quehacer que va más allá de administrar o "gerenciar" organizaciones, la innovación, la mejora continua, el liderazgo pedagógico o la visión de futuro. Así pues, la gestión en una organización educativa debe reconocerse como una serie de 
procesos prácticos, pero fundamentados en la teoría, que se han integrado horizontal y verticalmente para cumplir la multiplicidad de funciones que demanda la sociedad de una institución educativa de educación superior a distancia. El Instituto Internacional de Planeamiento de la Educación (IIPE) de la UNESCO define la gestión educativa como

(...) las acciones desarrolladas por gestores que pilotean amplios espacios organizacionales. Es un saber de síntesis capaz de ligar conocimiento y acción, ética y eficacia, política y administración en procesos que tienden al mejoramiento continuo de las prácticas educativas; a la exploración y explotación de todas las posibilidades; y a la innovación permanente como proceso sistémico (IIPE, 2000, p. 16).

La modalidad de una institución educativa a distancia, en línea o abierta, gestiona sus unidades y funciones de forma particular y específica; distinta a las universidades presenciales. En esta propuesta se presenta el detalle del ámbito de la gestión de este tipo de universidades, desde cuatro elementos que son; a saber, el recurso humano, la infraestructura física, el recurso financiero y el recurso tecnológico.

Al respecto, es sabido que los recursos como tales forman parte intrínseca de las necesidades de las entidades educativas y representan una parte fundamental del quehacer para la entrega de la docencia. Lo anterior es explicado por García Aretio (2014) de la siguiente manera:

La estructura organizativa y de gestión, igualmente, ha de adoptar un enfoque específico y muy diferente a los mantenidos tanto en la enseñanza presencial como en la más convencional enseñanza a distancia. Organización de las competencias y tareas docentes, gestión de las mismas, diseño del aprovechamiento de la plataforma o entorno utilizado, gestión del seguimiento personal, académico, administrativo, etc., y de la evaluación de los estudiantes, organización de los diferentes ámbitos de interacción, etc. En definitiva toda la organización de una institución docente debe estar enfocada hacia el estudiante, su rendimiento y satisfacción, así como al logro de los objetivos institucionales (p. 123).

A continuación, se explican los cuatro ejes de la gestión institucional: 


\section{- Recurso humano}

Desde la gestión, el recurso humano de la educación a distancia ha de concebirse como un grupo de actores que toman decisiones conjuntas continuamente, y que se relacionan entre sí desde diferentes rutas y vínculos. Se representa este recurso como un área fundamental pero particularmente diferente en relación con otras organizaciones de educación superior sobre todo, por las competencias que deben generar los tutores o docentes con base en las actividades atribuidas desde un entorno a distancia, virtual o en línea, poco conocidas por los profesores o facilitadores quienes en su mayoría han sido formados en entornos presenciales.

Desde esa misma perspectiva Lafourcade (2013, p. 44) señala que

La complejidad y cantidad de tareas que se cubren en una institución educativa, que esté siendo potenciada paulatinamente en su capacidad para alcanzar efectividad y productividad en los esfuerzos de logro de sus propósitos, exige la inclusión de personal técnico y administrativo idóneo para el desempeño de sus funciones. Complementariamente, requiere del equipamiento necesario.

En relación con lo anterior, la contribución del recurso humano, tanto en los aspectos administrativos como técnicos, se constituye en la condición necesaria para aumentar la probabilidad de que tales logros se obtengan.

El recurso humano, eje también conocido como los "personajes" son el componente decisivo para el desarrollo de una institución educativa de cualquier índole o nivel, a lo que la educación superior a distancia no se escapa. Para esta propuesta, se destacan entre estos actores:

Los usuarios quienes, se definen como las personas que "se benefician de la actividad o de los resultados del sistema" (Olivera, 2011, p. 191). Para este efecto los estudiantes; también nombrados como destinatarios (García, 2014, p. 45). Se les considera el recurso más importante en el acto educativo. Es la unidad básica hacia la que se dirigen todos los esfuerzos del proceso educativo y en función del cual se estructura todo el proceso. En una institución de educación superior ha de concebirse como el centro de todo el quehacer universitario lo que implica reforzar esfuerzos para familiarizarse con su desarrollo psicológico, su contexto social y ambiental, sus experiencias previas, su estilo de aprendizaje, sus motivaciones y sus 
expectativas o ambiciones profesionales, en fin conocer a quién va dirigido el servicio que presta la institución, con la finalidad de lograr un buen ejercicio de la labor de educar.

Como contraparte de este recurso se encuentran "los prestantes; o sea, los que desempeñan papeles activos, facilitan trabajos o servicios para fines del sistema."(Olivera, 2011p. 191).

Es el recurso humano que se aboca a la prestación de su trabajo para que el sistema funcione de manera óptima.

Específicamente, está representado por los docentes en quienes recae el proceso de enseñanza y quienes desempeñan un rol activo en la entrega del servicio a los destinatarios o usuarios. Su responsabilidad es tal que la eficiencia y la eficacia de las instituciones educativas, tanto a distancia como presenciales, obedece, en gran medida de las idoneidades y habilidades de los docentes o tutores.

Dentro del aparato organizacional de la institución educativa a distancia, ha de hacerse referencia a otros individuos que sostienen el engranaje del sistema y que le permiten su marcha, son también parte de los prestantes; llamados administrativos e investigadores, tales como asistentes universitarios, diseñadores de materiales escritos, administradores web y tantos otros funcionarios universitarios; son expertos que por su formación, actitudes y capacidades que responden a funciones muy distintas a las de una institución de educación superior presencial; manejan competencias específicas en espacios científicos, andragógicos o computacionales de diferentes áreas de la administración y la docencia, el diseño de cursos, la evaluación de los aprendizajes, la producción de materiales, la tecnología de la educación, el desarrollo profesional, etc.

\section{- Recurso tecnológico}

La tecnología aplicada a la educación, en el caso que atañe a este modelo, a distancia, abierta o en línea, se desarrolla dentro de un proceso complejo que permite articular diferentes recursos para ser facilitadores de los procesos de aprendizaje, como lo exponen Fallas y Trejos (2013) 
Los recursos para el aprendizaje son aquellos que se pueden utilizar en cualquier recurso tecnológico, el cual articula en un determinado sistema de símbolos ciertos mensajes con propósitos didácticos. El inmenso conjunto de recursos para el aprendizaje es una parte central del campo de la Tecnología Educativa.

La variedad de recursos -desde seres humanos, hasta herramientas, tecnologías y materiales diseñados para facilitar el aprendizaje- se ha incrementado de manera notable en los últimos años. Asimismo, el campo de la Tecnología Educativa se ha visto favorecido por las constantes innovaciones tecnológicas y por el desarrollo de la comprensión sobre cómo estas herramientas y recursos pueden favorecer al aprendiz (p. 58).

Agregan las autoras citadas anteriormente que "los recursos tecnológicos delimitan el ámbito de trabajo de la Tecnología Educativa, por ejemplo, vídeos, películas, programas de televisión, programas de audio, programas computacionales y telecomunicaciones, entre otros" (p. 58).

La evolución en el contexto educativo en una institución de educación superior a distancia, abierta o en línea, al encontrarse en el mercado alternativas innovadoras, debe ajustar constantemente la mediación entre la educación y el medio utilizado para un fin particular.

Sobre este aspecto, Ruiz, Gómez, Lara y Mestre (2013) mencionan que

La explicitación por parte de las instituciones de educación superior de las tecnologías disponibles en los procesos de formación a distancia es importante para garantizar la sostenibilidad y calidad de la misma, así como los requerimientos de usabilidad tecnológica por parte de los estudiantes. En cualquier caso, es importante caracterizar las tecnologías que se utilizan para el almacenamiento, circulación y acceso a los contenidos didácticos, los procesos de comunicación pedagógica, las operaciones académico administrativas y los soportes técnicos o informativos necesarios (p.64). 
Como parte de este recurso, es imprescindible referir la infraestructura digital; definida por Prieto J. (2013) "Esta variable se refiere a la disposición de los recursos tecnológicos necesarios que permiten a docentes y aprendices conectarse e interactuar, en un espacio idóneo de virtualidad" (p. 9).

\section{- Recurso financiero}

Es el recurso que permite darle continuidad en el tiempo a la institución y a los servicios que ofrece. El análisis de este recurso aclara las fuentes de financiamiento y la manera en que se presupuestan e invierten los recursos económicos en la universidad. Además, permite determinar el monto al que asciende el gasto institucional y la distribución de este en los múltiples débitos que tiene la institución.

\section{- Infraestructura física}

El estudio de la infraestructura comprende la descripción cuantitativa y cualitativa de las instalaciones y del equipamiento institucional. Es importante conocer los activos de la institución, determinar la cantidad y calidad de los equipos didácticos, de producción de materiales, de computación y otros propios de las acciones universitarias. Para Rojas (2010),

La administración de recursos físicos comprende todo un conjunto de aspectos relacionados con el uso eficaz y eficiente de terrenos, edificios, espacios, equipos, herramientas, mobiliario y material educativos, con el objeto de favorecer el desarrollo del curriculum, procurando conseguir los objetivos y metas del proceso educativo y los fines y propósitos del servicio educativo (p. 84).

Todos estos recursos mencionados, y otros particulares que cada institución educativa por comparar considere pertinentes, permiten el abordaje de una relación de semejanza y diferencia. Sin embargo, para finalidades de análisis, es necesario, al menos momentáneamente, aislar los recursos de su medio y manejar las variables. Luego se establecerá su relación con el sistema total.

Ahora bien, dado que el cambio continuo es la condición más regular en la sociedad actual, y este afecta todas las áreas del quehacer humano; la educación debe abrirse a estas nuevas 
formas de resolver, debe ajustar sus objetivos y debe modificar sus sistemas de administración, sus métodos, sus técnicas y todas aquellas otras tareas que la conforman, con el objetivo de estar en consonancia con las necesidades de los estudiantes y del momento histórico.

Esta propuesta de modelo para la investigación comparativa entre universidades de educación a distancia, abiertas o en línea, no pretende ser exhaustiva, sino que, por el contrario, busca permitir al investigador enriquecer su estudio, experiencia y creatividad. Es un modelo abierto y sensible a una constante revisión y reelaboración, dado que los entornos de los usuarios son heterogéneos. Asimismo, el modelo se crea en un contexto particular y único, dado que la UNED es la única institución de educación superior estatal a distancia en Costa Rica, y su patrón de relación se amplía a ámbitos internacionales y globales.

Para llevar a la práctica esta propuesta, es necesario aplicarlo como un modelo analíticodescriptivo apto que contiene ordenadamente toda la información y que permite trazar una lista de indicadores para la recolección de datos. No obstante, no se debe quedar ahí: es posible ampliarlo con modelos explicativos e interpretativos que guíen el trabajo a siguientes niveles.

El modelo se grafica de la siguiente forma:

\section{Figura 1.}

Modelo para la investigación comparativa entre universidades de educación a distancia, abiertas o en línea 


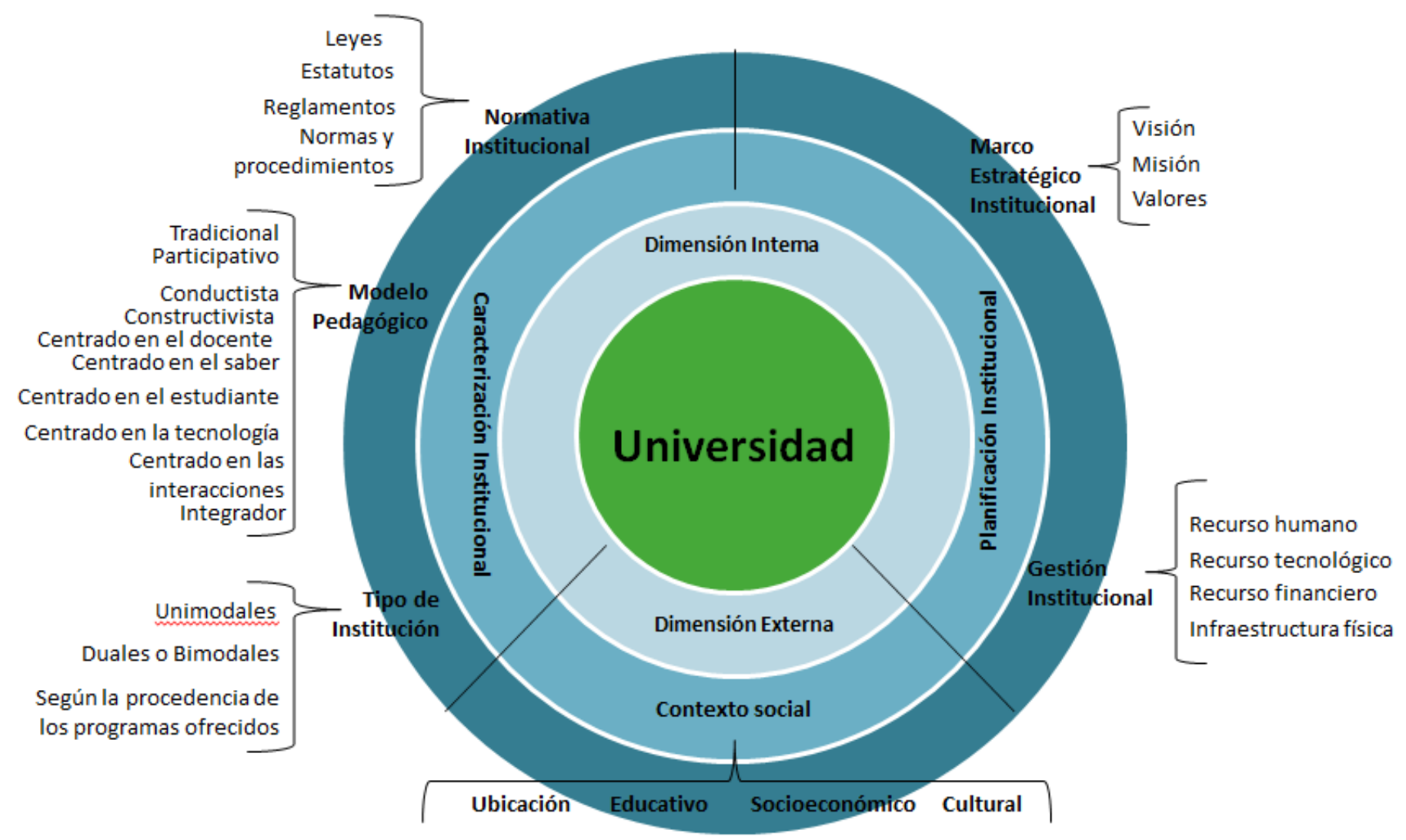

Fuente: Abarca, Azofeifa y Brenes (2015)

Para comprender la representación gráfica del modelo, se recomienda lo siguiente:

i. La figura del modelo se lee del centro hacia la periferia.

ii. Se articula de forma tal que cada sección se puede aplicar como herramienta de estudio particular o bien, aprovechar el modelo en su totalidad.

iii. Las representaciones de la periferia son las categorizaciones que requieren ser precisadas con mayor especificación.

iv. Como instrumento de trabajo en su totalidad permite realizar tres estudios simultáneos.

v. El modelo, aplicado a dos o más instituciones educativas a distancia, abiertas o en línea, como instrumento para estudios de educología comparativa, explicará las diferencias y las semejanzas entre ambos objetos confrontando elemento por elemento. 
Capacidad explicativa del modelo:

Cabe describir el modelo comparativo en lo que su diagramación representa; una figura circular que abarca cuatro planos o niveles correspondientes a los ejes prácticos en donde engrana el funcionamiento de la estructura educativa, se dispuso ésta forma geométrica, ya que permite dar sentido de organización, infinitud y conjunto. Cada plano se fracciona en diversos ámbitos del sistema; esto es:

- la Universidad: dispuesta en el círculo central, es el epicentro del modelo del que emana y a su vez, reúne en sí misma, las dimensiones y unidades que la conforman.

- Dos dimensiones, en el segundo nivel de la figura, representan el contexto interno y externo al sistema en estudio. Ambas dimensiones conforman el primer anillo en un color más claro:

- La dimensión externa: facilita al investigador acercarse al ambiente externo en el que se encuentra inmersa la institución educativa, describe aspectos que influyen sobre ella desde fuera.

\section{○ La dimensión interna:}

- Abarca la estructura organizativa formal

- El segundo anillo de color intermedio, está compuesto por tres grandes categorías ya descritas anteriormente:

- el contexto social

- la caracterización institucional

- la planificación institucional

- El tercer anillo del modelo que se aprecia de color más oscuro en el plano externo, detalla cada una de las categorías propuestas en el segundo anillo. 
- La representación de la ubicación geográfica - espacial, el contexto educativo, socioeconómico y cultural permite examinar los elementos que, desde la sociedad, se relacionan con la institución educativa pero a su vez, la determinan; estos elementos completan la descripción del contexto social.

- La normativa, el modelo pedagógico y el tipo de institución pretenden puntualizar la caracterización de las universidades por comparar.

- En el mismo nivel de especificación; tanto el marco estratégico institucional como la gestión institucional especifican la categoría que refiere a la planificación institucional.

\section{Conclusiones y recomendaciones}

\subsection{Conclusiones}

Realizar una comparación válida necesariamente se basa en información relativa a dos o más objetos que se desean comparar, ya sean los sistemas completos, educativos en este caso; o sectores, problemas o fenómenos, expresados en términos de definiciones y categorías que comparten. Los datos obtenidos a partir de la aplicación de este instrumento permitirán realizar una descripción y análisis profundo y simultáneo entre sistemas, lo que resaltará sus semejanzas y diferencias.

Este modelo establece una correlación entre el sistema y el entorno externo que lo determina, de manera que es un modelo abarcador, ya que visualiza todo el sistema desde una perspectiva macro, y no solo estudia los aspectos de orden pedagógico que tradicionalmente son el objeto material de investigaciones en estas instituciones.

La propuesta en discusión está construida en estrecha relación con las teorías de gestión y organización actuales, y se vincula a los requerimientos de la época. La aplicación de este instrumento facilitará la dirección del cambio organizacional en los sistemas, así como el mejoramiento de la gestión en las universidades de educación a distancia, abierta o en línea. En este sentido, el modelo no solo es aprovechable para las necesidades de descripción, sino que también funciona como una guía para la explicación e interpretación de la realidad. 
Inclusive, es posible utilizar el modelo como instrumento de diagnóstico institucional en los ámbitos señalados, por contar con una estructura abarcadora, abierta y flexible.

Este modelo surge a partir de la práctica fundamentada en una trayectoria madura de más de 35 años de la UNED Costa Rica; lo que permite y exige este tipo de procesos para aportar conocimiento y al mismo tiempo, intenta acercar otras realidades y experiencias en el ámbito de la educación superior a distancia, abierta o en línea. Igualmente, el modelo procura generar un impacto real en investigaciones o estudios formales y dejar de lado la especulación, de forma tal que contribuya a la mejora de los sistemas educativos a través del mundo. Por último, es de esperar que este modelo posibilite el contacto con redes flexibles internacionales para promover la innovación de la modalidad educativa a distancia, abierta o en línea.

\subsection{Recomendaciones}

Se sugiere la aplicación y validación del presente modelo en futuras etapas de investigación, con la finalidad de enriquecer los procesos de conducción de los sistemas educativos a distancia, abierta y en línea, para que la toma de decisiones pueda apoyarse en resultados científicos confiables. Dicha validación permitirá, en primer lugar, darle continuidad y alcance al cuarto objetivo específico planteado en la propuesta de investigación; y en última instancia, ejecutar el modelo en la práctica investigativa.

Según lo anterior, se propone a corto plazo, definir instrumentos y elaborar los indicadores representativos de cada nivel; así como iniciar la validación para profundizar en la descripción, mediante la propuesta de un estudio de caso.

\section{Referencias}

Abarca, A., Azofeifa, J. y Brenes, A. (2015) Informe Modelo para la Investigación Comparativa entre Universidades de Educación a Distancia, Abiertas o en Línea. San José, Costa Rica: EUNED.

David, F. (2008). Conceptos de Administración Estratégica. Ciudad de México, México: Pearson Educación. 
Definición.DE. (2015). Definición de modelo. Recuperado de http://definicion.de/modelo-degestion/

Fallas, I. y Trejos, I. (2013). Educación en la sociedad de la información y el conocimiento. San José, Costa Rica: EUNED.

García Aretio, L. (2004). Viejos y nuevos modelos de educación a distancia. Recuperado de: http://www.researchgate.net/publication/39214322

García, L. (2014). Bases, mediaciones y futuro de la educación a distancia en la sociedad digital. Madrid, España: SíNTESIS.

González, Nieto, Portela y Martínez. (2012). Administración de la educación. Estado de México, México: Pearson.

Instituto Internacional de Planeamiento de la Educación (IIPE). (2000). Gestión Educativa Estratégica, Módulo 2. Buenos Aires, Argentina: UNESCO.

Lafourcade, P. (2013). Evaluación Institucional. San José, Costa Rica: EUNED.

Lobo, N., y Fallas, V. (2008). La Benemérita Universidad Estatal a Distancia en la Sociedad del Conocimiento. San José, Costa Rica: EUNED.

Moreno, M. (2012). Veinte visiones de la educación a distancia. México: UEDGVIRTUAL.

Olivera, C. (2011). Introducción a la Educación Comparada. San José, Costa Rica: EUNED.

Prieto J. (2013). Una aproximación metodológica al uso de redes sociales en ambientes virtuales de aprendizaje para el fortalecimiento de las competencias transversales de la Universidad EAN. Virtu@Imente, 1, (1). 1-16. Recuperado de: http://journal.ean.edu.co/index.php/vir/article/view/1397

Real Academia Española. (2014). Diccionario de la lengua española (23 ed.). Madrid, España. Recuperado de http://dle.rae.es/?id=PTk5Wk1

Rojas, C. (2010). Administración de recursos físicos en educación. San José, Costa Rica: EUNED.

Ruiz, E., Gómez, J., Lara, J., y Mestre, G. (2013). Modelo de educación a distancia. Universidad Tecnológica de Bolívar. Recuperado de: http://www.unitecnologica.edu.co/educacionadistancia/sites/default/files/Investigaci\%C 3\%B3n-\%20Documento\%20Creaci\%C3\%B3n\%20CEaD\%20UTB.pdf 
Saba, F. (2014). Methods of Study in Distance Education: A Critical Review of Selected Recent Literature. En: Zawacki-Richter, O. y Anderson, T. (Ed.), On Line distance Education: Towards a Research Agenda (pp. 151-171). Alberta, Canadá: AU Press.

Senge, P. (2011). La quinta disciplina. El arte y la práctica de la organización abierta al aprendizaje. Buenos Aires, Argentina: Granica.

UNESCO. (2015a). Definición de Educación. Recuperado de: http://www.uis.unesco.org/Pages/Glossary.aspx

UNESCO. (2015b). Definición de Programas de educación a distancia que son habilitados por las TIC. Recuperado de http://www.uis.unesco.org/Pages/Glossary.aspx

Zawacki-Richter, O., y Anderson, T. (2014). On Line distance Education: Towards a Research Agenda. Alberta, Canadá: AU Press. 